This item was submitted to Loughborough's Research Repository by the author.

Items in Figshare are protected by copyright, with all rights reserved, unless otherwise indicated.

\title{
Defect generation and pileup of atoms during nanoindentation of Fe single crystals
}

PLEASE CITE THE PUBLISHED VERSION

PUBLISHER

(C) American Physical Society

LICENCE

CC BY-NC-ND 4.0

REPOSITORY RECORD

Smith, Roger, D. Christopher, Steven D. Kenny, A. Richter, and B. Wolf. 2019. "Defect Generation and Pileup of Atoms During Nanoindentation of Fe Single Crystals". figshare. https://hdl.handle.net/2134/1743. 


\title{
Defect generation and pileup of atoms during nanoindentation of Fe single crystals
}

\author{
Roger Smith, D. Christopher, and S. D. Kenny \\ School of Mathematics and Physics, Loughborough University, Leicestershire, LE11 3TU, United Kingdom
}

Asta Richter and Bodo Wolf

Department of Engineering Physics, University of Applied Sciences Wildau, Bahnhofstrasse, D-15745 Wildau, Germany

(Received 11 December 2002; revised manuscript received 10 March 2003; published 6 June 2003)

\begin{abstract}
Complementary large scale molecular-dynamics simulations and experiments have been carried out to determine the atomistic mechanisms of the nanoindentation process in single crystal $\mathrm{Fe}\{110\},\{100\}$, and $\{111\}$. The defect formation and motion causes the complex mechanisms of plastic and elastic deformation which is reflected in the pileup patterns. The experimental results show distinct patterns of pileup material which are dependent on the individual crystal faces and the superposition of the stress field of the indenter. The highest pileup around the indenter hole occurs on the $\{100\}$ surface and the shallowest on $\{111\}$. The least symmetric surface is $\{110\}$ which produces an experimental pileup pattern displaying only twofold symmetry with the axially symmetric indenter. The pyramidal indenter produces an asymmetric pattern which changes as the crystal is rotated with respect to the tip but repeats with threefold rotational symmetry. Material displacement occurs primarily in planes of the $\{110\}$ family. Pileup is formed by cross slip between planes of the same family which intersect in $\langle 111\rangle$ directions. For the $\{110\}$ surface, dislocation loops propagate in the four in-plane $\langle 111\rangle$ directions and the two inclined $\langle 111\rangle$ directions. The loops that propagate in the in-plane directions are terminated by edge dislocations at the surface. These transport material away from the tip but cannot produce pileup. The loops that propagate in the inclined direction cross slip and cause the observed pileup. The $\{100\}$ surface has fourfold rotational symmetry and all the $\langle 111\rangle$ directions are inclined. The dislocation loops propagate in these directions and cross slip readily occurs, leading to a large pileup. The $\{111\}$ face shows the least pileup which is more spread out over the surface. In this case the dislocation loops propagate in shallow slip planes and do not readily cross slip. Experimentally determined force-depth curves show distinct "pop-ins" which correspond to the formation of dislocations. The contact pressure (nanohardness) is not a constant and increases with decreasing indentation depth. It also changes with crystal face. Calculated force-depth curves match the experimental trend but give estimates of the nanohardness and Young's modulus higher than those values experimentally determined.
\end{abstract}

DOI: 10.1103/PhysRevB.67.245405 PACS number(s): 68.55.Ln, 61.72.Bb, 61.72.Ff, 68.35.Gy

\section{INTRODUCTION}

Nanoindentation ${ }^{1-4}$ has become an established technique for determining the mechanical properties of surfaces and thin films on a scale for which the fate of individual atoms is important. As nanoindentation becomes a standard engineering tool, it is useful to establish a connection between the atomic scale processes during the indentation and the measurable mechanical properties. The usual mechanical properties that are determined during nanoindentation are the Young's modulus and the "nanohardness" of the material. These are obtained from an analysis of the dynamic relationship between applied force and indentation depth. An advantage of the technique is that it can be combined with scanning force microscopy so that an image of the probed area can be obtained with the same tip as is used for the indentation. The method has many potential applications for examining nanoscale mechanical properties of small areas and thin films.

However, although the method has this potential to determine properties of systems of complex materials, the atomic scale mechanisms underlying the method are not well understood. These can be better studied when well-characterized single crystal samples are investigated since the mechanisms involved are fewer. In this paper complementary experiments and molecular dynamics (MD) simulations are carried out on the $\{110\},\{100\}$, and $\{111\}$ faces of single-crystal Fe to investigate the atomic scale processes and the defect formation and pileup around the indenter hole. A similar experimental study without simulations has been undertaken with singlecrystal $\mathrm{Au},{ }^{3}$ where the crystal anisotropy for an fcc lattice was investigated. Gold has also been studied using MD where dislocation loops have been shown by simulations to propagate a considerable distance from the indentation region. ${ }^{5}$ Indeed much of the understanding of the nanoindentation process has come about by a combination of scanning probe microscopy with classical and quantum moleculardynamics simulations. ${ }^{5-9}$ The simulations have shown how elastic deformation can occur, ${ }^{6}$ dislocations can propagate, ${ }^{5,10,11}$ and the way in which pileup around the indentation hole ${ }^{7}$ is formed.

The deformation and defect propagation that occurs in crystals is anisotropic and when combined with a nonaxially symmetric indenter can induce a complicated overlap of mechanisms. It is this overlap between indenter geometry and crystal symmetry that is one of the aims of the present investigation. 


\section{SAMPLE PREPARATION AND OPERATIONAL PROCEDURE}

Iron single crystals were grown in rods and sliced into discs of 3-mm thickness, then oriented by x-ray diffraction, and polished using an aluminum and diamond suspension down to a $0.25-\mu \mathrm{m}$ finish, as in standard metallographic techniques. All nanoindentation experiments were performed using the electrostatic transducer of the Hysitron triboscope attached to a scanning force microscope (SFM), the Nanoscope III. The transducer consists of a three-plate capacitor, the midplate of which carrying the impression tool fixed to a thin stylus. Application of a dc voltage generates an electrostatic force driving the indenter into the sample surface. Simultaneously the capacity change as a measure of penetration depth is recorded resulting in a force-depth curve. The indenter can be used for scanning over the sample surface for imaging. For further details see Refs. 12-14. Combining nanoindentation with surface imaging provides access to the imprint topography from which information on preferred material transport directions, formation of piling-up or sinkingin, and crack generation and propagation in the case of less ductile materials can be gained.

\section{EXPERIMENTAL RESULTS}

The distribution and symmetry of piling up should be a reflection of the local crystalline orientation. Indenters have a special geometry which overlaps with the crystal symmetry. However, nanoscale cones are difficult to manufacture, hence pyramids are commonly preferred in indentation testing. In comparison to spheres the pyramids have the further advantage of self-symmetry, i.e., the ratio between lateral and depth extension of the generated imprint is independent on load. In the case of mesoscopic and macroscopic indents with imprint sizes larger than distances between hardness governing defects (dislocations, stacking faults, grain boundaries) this self-symmetry gives rise to constant hardness. ${ }^{15}$ In nanoindentations, however, impression bodies must always be considered as blunted or truncated. Furthermore, the imprint size is smaller or comparable to the length scale of defects. As a consequence the hardness changes with load and impression size, even if the purely geometric deviation from an ideal pyramid indenter shape is small. (This can be seen later in Fig. 9 below.) Apart from a diamond spherocone indenter [Fig. 1(b)], three-sided pyramids (cube-corner indenters) were used in this work. In isotropic materials, e.g., glass, the occurring pileup shows also a threefold symmetry with about the same height of all three hillocks. With such pyramidal indenters the induced stress is a maximum over the faces of the indenter and a minimum along the edges. The combination of the three-fold indenter symmetry, asymmetric stress distribution, and the crystal structure complicates the analysis of the SFM images. It is the relationship between crystal orientation, defect generation, and asymmetric pileup which is considered here. Separate faces of singlecrystal $\mathrm{Fe}$ are investigated. The most detailed results are for the $\{110\}$ and $\{100\}$ surfaces where the asymmetry effects of the indenter orientation are strongest. A few results have also been obtained for the $\{111\}$ crystal plane where the piling up (a)

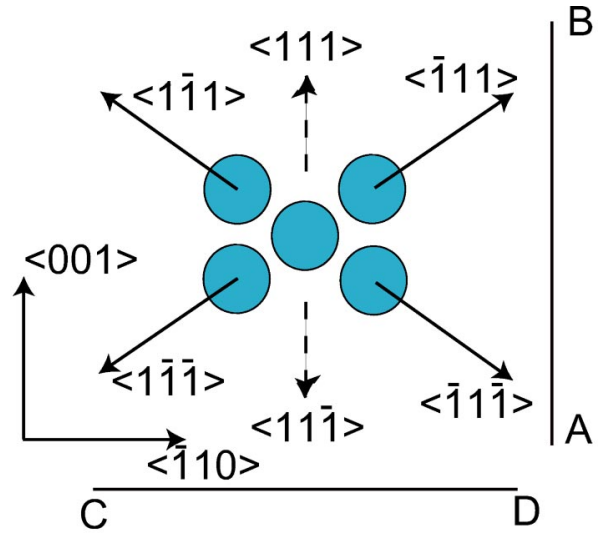

(b)

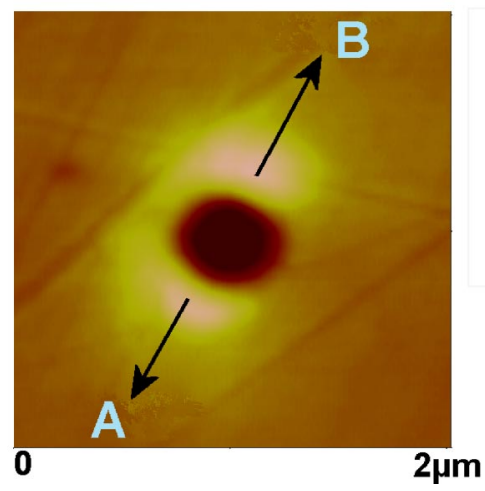

FIG. 1. (a) Schematic diagram of the arrangement of atoms in the $\mathrm{Fe}\{110\}$ surface; (b) the imprint left by a spherocone in the surface, together with the surface alignment indicated in (a). The direction of maximum pileup is marked by the line $\mathrm{AB}$.

adjacent to the different indenter faces appears very similar irrespective of its orientation. The pileup patterns can be also affected by a misorientation between the sample normal and the tip axis. However, we have not investigated this effect and for the purposes of this study it is assumed that all the samples are correctly aligned.

\section{A. Results for the $\{110\}$ plane}

The arrangement of atoms in the surfaces is shown schematically in Fig. 1(a) together with the two axes of reflection symmetry, $\mathrm{AB}$ and $\mathrm{CD}$, for the $\{110\}$ surface. The in-plane $\langle 111\rangle$ directions are marked with solid lines crossing at an angle of $70^{\circ}$. The out-of-plane $\langle 111\rangle$ directions are marked with a dashed line. Using a rotationally symmetric spheroconical indenter, the piled-up material around the indenter exhibits a twofold symmetry [Fig. 1(b)]. In Fig. 1(b) a line $A B$ is shown, drawn through the center of the piled-up material and this marks the out-of-plane $\langle 111\rangle$ direction as shown in Fig. 1(a). The crystal directions were directly measured using a scanning electron microscope in combination with Kossel techniques.

In reality the pyramidal indenter is not atomically sharp and ours has a round apex of about $100 \mathrm{~nm}$, obtained by a calibration grid. ${ }^{6}$ Figure 2 shows how the imprint shape and the pileup changes with increasing load. Note that the same tip is used for imaging as for indenting so that whereas the 


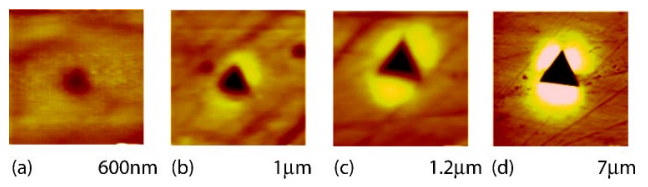

FIG. 2. A series of scanning force images with cube-corner pyramidal indenter imprints on the $\mathrm{Fe}\{110\}$ surface as a function of increasing load: (a) maximum force $F=0.1 \mathrm{mN}$, maximum height $40 \mathrm{~nm}$; (b) $F=0.2 \mathrm{mN}$, maximum height $60 \mathrm{~nm}$; (c) $F=0.4 \mathrm{mN}$, maximum height $100 \mathrm{~nm}$; (d) $F=6 \mathrm{mN}$, maximum height $200 \mathrm{~nm}$.

heights above the original surface are accurately given, the tip cannot scan exactly and measure accurately the depth of the indentation hole. For very small loads up to $0.1 \mathrm{mN}$, Fig. 2(a) shows that no pileups can be imaged using the SFM, and the indenter cross section is almost axially symmetric. As the load increases to $0.2 \mathrm{mN}$, two symmetric pileups occur as for the spherocone, with Fig. 2(b) depicting the specific symmetry line. At a load of $0.4 \mathrm{mN}$, the asymmetries due to the pyramidal indenter shape begin to appear, Fig. $2(\mathrm{c})$. However, when the load is increased to $6 \mathrm{mN}$ an asymmetric piling up around the three-sided imprint is seen, Fig. 2(d). The investigation of the piling-up pattern is considered in more detail in Fig. 3.

First the cube-corner diamond indenter is aligned along the $A B$ axis which is shown in Fig. 1(b). Figure 3(a) shows the result of the cube-corner indentation using this orientation. Instead of two symmetrical pileups, material piled up along each side of the triangle is visible. More material is piled up along the edge of the triangle that is perpendicular to the line $\mathrm{AB}$ with two smaller pileups on the other sides of the triangle. The height of the largest hillock is $82 \mathrm{~nm}$ whereas the other two have almost equivalent heights of 39 $\mathrm{nm}$ and $43 \mathrm{~nm}$, respectively, in accordance with the sample symmetry. In this figure the length of the indenter side is $0.73 \mu \mathrm{m}$. This picture appears since the two pileups corresponding to the crystal symmetry are split into different areas guided by the indenter geometry. For an isotropic material, the stress field induced by the cube-corner indenter is not homogeneous, as in the case of a spherocone, but induces a larger stress over the pyramidal surface area (edges of the triangle) than along the edges of the pyramid (corners of the triangle). In Figs. 3(a) and 3(b) the arrows and numbering

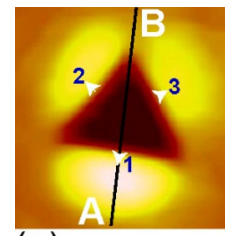

(a)

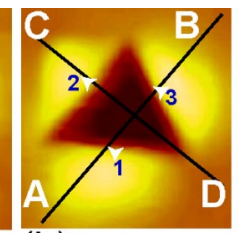

(b)

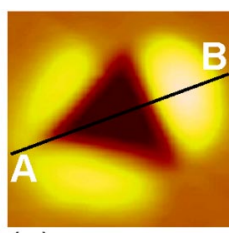

(c)
FIG. 3. Scanning force images after indentation by a cubecorner pyramidal indenter on $\mathrm{Fe}\{110\}$ as a function of the azimuthal surface orientation. The marked axis $\mathrm{AB}$ is in the same direction as that in Fig. 1. (a) corresponds in our notation to a rotation angle of $0^{\circ}$ and shows reflection symmetry about the line $\mathrm{AB}$. After rotation of the crystal through $30^{\circ}$ the imprint shown in (b) is obtained with a reflection symmetry line $\mathrm{CD}$; (c) after a rotation of $60^{\circ}$. The maximum height scale in all images is $130 \mathrm{~nm}$. refer to the directions of maximum stress along each face of the pyramid. Thus larger hillocks appear if the preferred crystal direction for pileups coincides with an aligned pyramidal side of the cube-corner indenter. The larger pileup occurs with exactly the same position as that for the spherocone. The second hillock along the preferential crystallographic axis for pileups splits into two smaller hillocks. These two different pileup areas are separated by a line which passes through the center of gravity of the indent image triangle and is perpendicular to the marked crystallographic axis $\mathrm{AB}$. That type of pileup pattern is repeated after rotating the sample surface by $60^{\circ}$ with respect to the fixed indenter [see Fig. 3(c)]. Rotation is achieved by means of a table which can be moved with a step motor in the $x, y$, and $z$ directions by manual operation to bring the sample in the desired position. This table can be also turned in the $x-y$ plane by fine tuning screws. In order to perform definite conditions for adjustment of the sample before the indentation, an additional measuring scale has been constructed so that the sample could be adjusted to the fixed position of the indenter by manual operation with an accuracy of about $0.5^{\circ}$. In the case of rotation through $30^{\circ}$, Fig. 3(b), there is approximate reflection symmetry about the line $\mathrm{CD}$ with slightly larger pileup areas along the edges closest to the letters $\mathrm{C}$ and $\mathrm{D}$ on the diagram. In this figure twice the force was used as in Fig. 3(a) and the length of the indenter side is $1.2 \mu \mathrm{m}$. The highest pileup, close to the letter $\mathrm{B}$, has a height of $102 \mathrm{~nm}$ and the other pileups are smaller at $91 \mathrm{~nm}$ and $86 \mathrm{~nm}$. After rotation through $60^{\circ}$, the asymmetry in the pileup is again visible except that it is now rotated through that same angle.

\section{B. Results for the $\{100\}$ plane}

Figure 4 shows typical SFM images of two different orientations of indentation of the $\{100\}$ surface rotated through $45^{\circ}$. In Fig. 5(a) we see two large pileups (44-nm and 35-nm high) and one smaller one $(5 \mathrm{~nm})$ whereas in Fig. 5(b) we can see one large pileup (49 nm) and two smaller ones (22and 26-nm high). We can expect the maximum pileup to occur when the perpendicular to the pyramid edge was aligned with the $\langle 110\rangle$ direction and the least when the perpendicular is aligned along the $\langle 100\rangle$ direction. This is the explanation for the differences between the two figures. The two large hillocks on both sides of the $\langle 100\rangle$ direction and the two small ones on the left and right from $\langle 110\rangle$ should have equivalent heights. The deviations are due to lateral misalignment of the indenter (about $4^{\circ}$ ) and surface misorientation (about $5^{\circ}$ ).

\section{Results for the $\{111\}$ plane}

Figure 5 shows an SFM image of indentation of the $\{111\}$ surface with a force of $2 \mathrm{mN}$, which is a larger force than that used in Figs. 3 and 4. The piling-up is less asymmetric in comparison to the piling up pattern in the crystallographic planes $\{110\}$ and $\{100\}$. The maximum peaks are $55 \mathrm{~nm}, 25$ $\mathrm{nm}$, and $38 \mathrm{~nm}$. In this case the piled up material is spread out over a larger part of the surface than that for the $\{100\}$ 
(a)

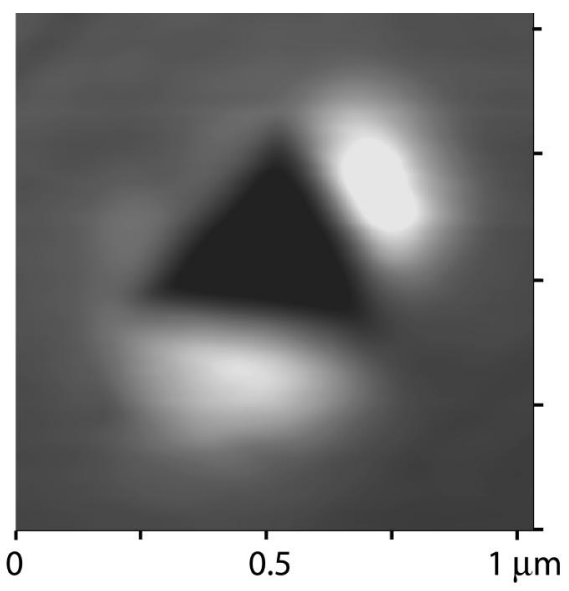

(b)

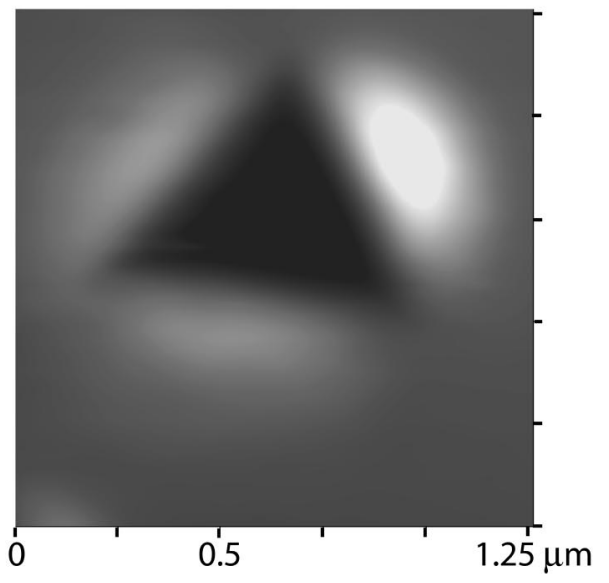

FIG. 4. Typical scanning force images of indentation by a cubecorner pyramidal indenter on $\mathrm{Fe}\{100\}$ as a function of the surface orientation; maximum force $F=1 \mathrm{mN}$ and maximum heights scale as (a) $80 \mathrm{~nm}$ and (b) $180 \mathrm{~nm}$, respectively.

plane and has approximately the same height as that for the $\{100\}$ plane but with double the indenter force.

\section{Force-depth curves and their analysis}

Figure 6 shows the ratio of the relative scales of the pileup material (measured by its maximum height) and the depth of the indent, measured for the $\{110\}$ and $\{100\}$ surfaces. For $\mathrm{Fe}\{110\}$ this ratio increases to a constant value for depths for which the influence of the rounded tip is no longer significant. This occurs at a load of $0.7 \mathrm{mN}$ and a depth of about $200 \mathrm{~nm}$. This implies that there is geometric selfsymmetry between the material pileup and the indentation hole for depths greater than about twice the radius of the rounded end of the pyramidal tip. For smaller indentation depths, the piled-up material is proportionally less, implying either a densification of the material or material transport away from the environs of the hole. For $\mathrm{Fe}\{100\}$ the pileup ratio decreases with increasing force and depth. The differences between the piling up on the two faces will be discussed later in the simulation section.

The load-depth curves for indentation into $\mathrm{Fe}\{110\}$ and $\mathrm{Fe}\{100\}$ by a cube-corner pyramidal indenter for large and

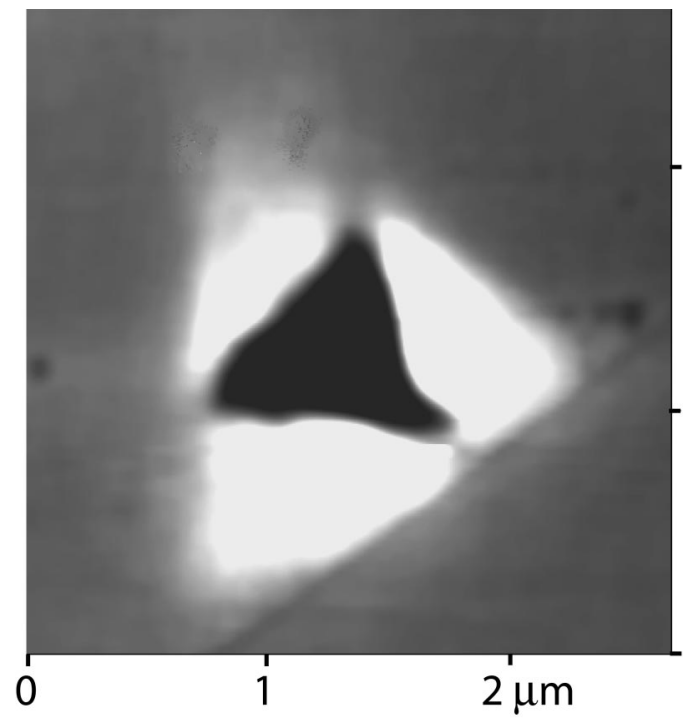

FIG. 5. A scanning force image of pileup on the surface of $\mathrm{Fe}\{111\}$ after indentation by a cube-corner pyramidal indenter. Maximum force $2 \mathrm{mN}$; maximum height $50 \mathrm{~nm}$.

small forces are shown in Fig. 7. The load-depth curve for the force of $0.9 \mathrm{mN}$, Fig. 7(a), shows a smooth form, characteristic of plastic deformation. Figure 7(b) shows the loaddepth curve for the smaller force of $0.18 \mathrm{mN}$. On this more detailed scale, the curve does not appear smooth but contains "pop-ins" marked by the arrows on the diagram at which the load remains constant as the depth increases stepwise. The pop-ins can be caused by a number of different mechanisms, including the failure of a thin oxide layer or the propagation of dislocations and point defects from under the tip. The propagation can be affected by asymmetries and asperities on the tip. One mechanism for the origin of these pop-ins will be discussed in the simulation section. It should be noted that the pop-ins occur only for small depth indentation and are not visible when the deeper portion of FIg. 7(a) is viewed with the same axes scaling as that in Fig. 7(b).

The contact pressure (hardness) is shown in Fig. 8 as a function of contact depth measured during indentation by a

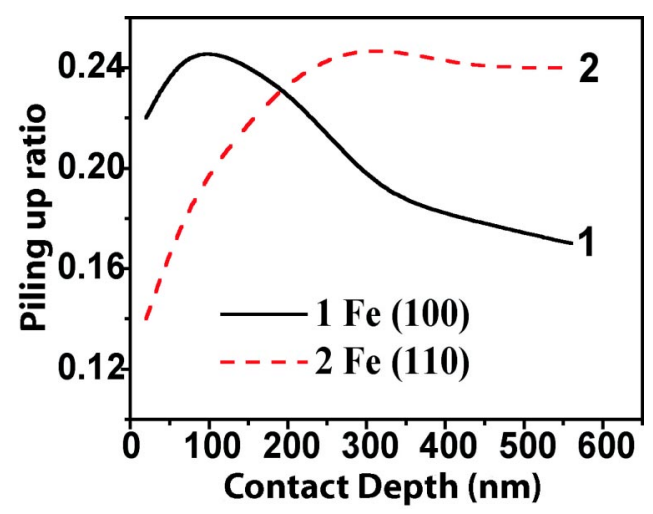

FIG. 6. The piling-up ratio, $h_{p} / h_{c}$, as a function of contact depth for $\mathrm{Fe}\{110\}$ and $\mathrm{Fe}\{100\}$ after indentation by a cube-corner pyramidal indenter. Here $h_{p}$ is the maximum pileup height and $h_{c}$ the contact depth. 

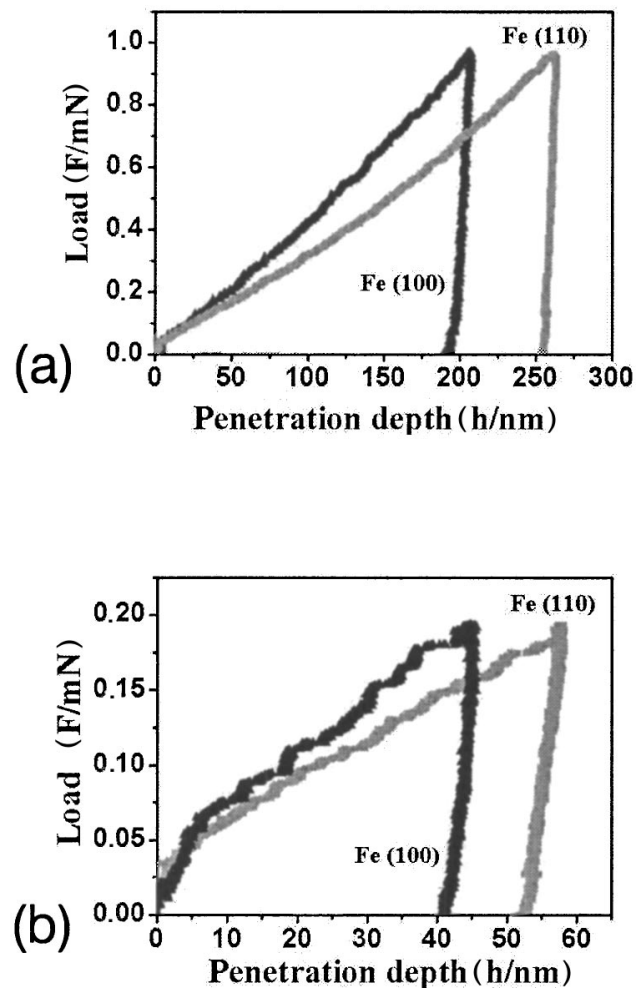

FIG. 7. The load-depth curves for indentation into $\mathrm{Fe}\{110\}$ and $\mathrm{Fe}\{100\}$ by a cube-corner pyramidal indenter for forces up to (a) 1 $\mathrm{mN}$ and (b) $0.2 \mathrm{mN}$.

pyramidal cube corner for the $\mathrm{Fe}\{110\}$ and $\{100\}$ faces. The differences in hardness between the two samples reflect both hardness anisotropy between the two faces and also possible differences in sample composition since the samples originated from different batches of growth. The results reflect the well-known situation in which the contact pressure decreases as the relative contribution to the hardness from intrinsic defects in the sample becomes more important than the elastic deformation of the material and the tip-induced generation of dislocations. For sufficiently large indentation depths the hardness reaches a constant value of $4.1 \mathrm{GPa}$ and 2.2 GPa for $\{100\}$ and $\{110\}$ faces, respectively.

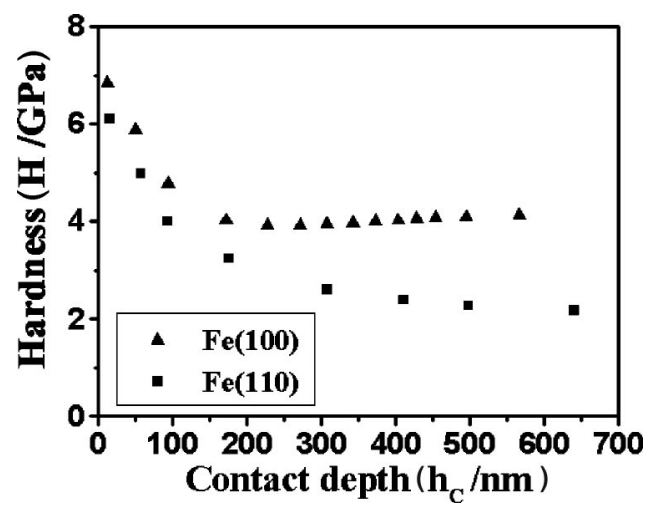

FIG. 8. Hardness as a function of contact depth measured during indentation by a pyramidal cube corner for the $\mathrm{Fe}\{110\}$ and $\{100\}$ faces. The loads varied between 0.05 and $6 \mathrm{mN}$.

\section{SIMULATION TECHNIQUES}

The simulated indenter has the configuration of a $90^{\circ}$ pyramid, and is formed by taking a cubic diamond crystal with $\{100\}$ faces and cutting along the diagonal $\{111\}$ plane. The most stable configuration occurs when the atoms on the three $\{100\}$ planes are dimer reconstructed. Experimentally the tip is not atomically sharp and so curvature is also added to the simulated tip, but the tip is still sharper than in the experiment when the ratio (radius of curvature)/(maximum penetration) is considered. The indenter used here is configured from 64373 atoms, with 5184 atoms forming the fixed top $\{111\}$ layers. The pyramid was blunted by removing the top $1.3 \mathrm{~nm}$ of the sharp end of the tip and adding a curved apex whose radius was $9 \mathrm{~nm}$. One simulation for the $\{110\}$ surface was also carried out with an infinitely hard paraboloidal interface with a tip radius of $2.5 \mathrm{~nm}$. The indenter is oriented so that the tip moves with the $\{111\}$ planes parallel to the surface of the sample. The indenter apex is positioned outside the range of the tip-substrate interaction potential at a height above the center of the substrate surface, where the indentation is to be made. The top three layers (i.e., the $\{111\}$ planes) of the indenter are constrained to move in a prescribed manner. The movement of the rest of the tip and the substrate is determined by integrating the equations of motion numerically. During each time step a sinusoidal displacement, $r(t)$, in the direction normal to the surface, is imparted to the fixed atoms described by

$$
r(t)=r_{\max } \sin (\pi t / T),
$$

where $r_{\max }$ is the maximum displacement of the rigid atoms, $T$ is the indentation period, and $t$ is the time elapsed. At time $t=0$, all the indenter atoms have the assigned velocity $r_{\max } \pi / T$. The indentation depth is measured by calculating the distance between the tip-terminating central carbon atom and the undisturbed substrate surface. The force acting on the indenter is evaluated by summing all the vertical force components on each atom of the indenter. The C-C interactions are described by Brenner's many-body potential ${ }^{16,17}$ smoothly cutoff at $2.0 \AA$. The $\mathrm{Fe}-\mathrm{Fe}$ interactions are described by the Finnis-Sinclair potential which is smoothly cutoff at $3.62 \AA$. The interaction between the indenter and the substrate atoms is assumed to be purely repulsive and is given by the Ziegler-Biersack-Littmark potential, ${ }^{18}$ smoothly cutoff at $3.59 \AA$. The reason for this simplification is that without an adsorbate cover, diamond is very reactive and the adhesive interactions with $\mathrm{Fe}$ are very strong and cause the tip to break on extraction. Previous simulations, which include passivating the diamond indenter surface with hydrogen, ${ }^{19}$ have shown that the use of a purely repulsive potential does not affect the formation of defects in the substrate or the force-depth curves during compression but does have some effect at the point of extraction of the indenter when the stress becomes tensile.

The substrates employed here take the form of a rectangular crystal lattice, with the indenting surface being approximately square. Periodic boundary conditions are applied to all sides in the horizontal plane. Each substrate has approximate dimensions $30 \mathrm{~nm} \times 30 \mathrm{~nm} \times 15 \mathrm{~nm}$, and for 


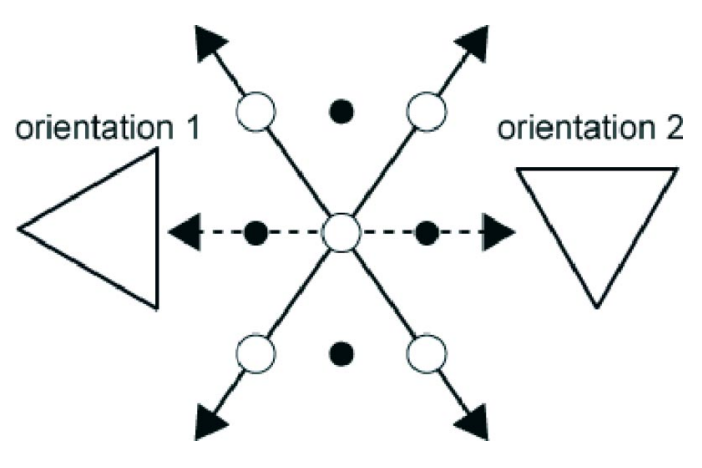

FIG. 9. The two tip orientations used in the simulation of indentation into the $\{110\}$ surface with respect to the layout of atoms in the surface layers. The open circles refer to the positions of atoms in the top layer and the filled circles to the second-layer atoms. The unbroken line arrows are the in-plane $\langle 111\rangle$ directions and the broken line arrows the out-of-plane $\langle 111\rangle$ directions.

each simulation on the three separate faces the entire system contained approximately 1.2 million atoms. Atoms on the horizontal edge of the substrate, and those composing the underside two layers, are fixed to prevent the substrate from displacing vertically during indentation. In order to carry out the calculations on a reasonably sized system, tip speeds faster than experiment have to be used. Tests with different tip speeds were carried out and the speed reduced until very similar calculated force-depth curves resulted. This resulted in an indentation period of $T=90 \mathrm{ps}$ with $r_{\max }=4.4 \mathrm{~nm}$. Since the simulated indentation speed is faster than experiment we adopted a quasistatic approach in which all atoms are damped to remove the excess energy imparted by the indenter. For simplicity the Lindhard-Scharff inelastic loss damping model is used to extract this excess energy from the system. ${ }^{7}$ The classical equations of motion for all atoms are integrated with a constant time step of $1.0 \mathrm{fs}$ using the velocity-Verlet algorithm. ${ }^{20}$ One simulation was also carried out using a parabolic interface model with a radius of curvature of $2.5 \mathrm{~nm}$ in order to model the axial symmetry of the spherocone. For the interface model, the tip is not treated atomistically. The force on the substrate atoms depends only on distance of the atom from the nearest point on the moving interface.

\section{SIMULATION RESULTS}

\section{A. Results for the (110) plane}

Because of the relationship between the crystal symmetry and the indenter geometry, two different rotation angles are employed, as illustrated in Fig. 9, referred to as orientation 1 and orientation 2, and corresponding to the orientation for the micrographs shown in Figs. 3(a) and 3(b), respectively.

Figure 10 schematically shows the direction of the piling-up formation for an axially symmetric indenter together with the layout of atoms in the surface. The $\langle 111\rangle$ directions along which pileup occurs are the lines of intersection of the $\{110\}$ slip planes and the surface. The details of this piling-up process are discussed further below. Figure 11 shows the surface topography after extraction of the indenter. The paraboloidal indenter exhibits twofold reflection

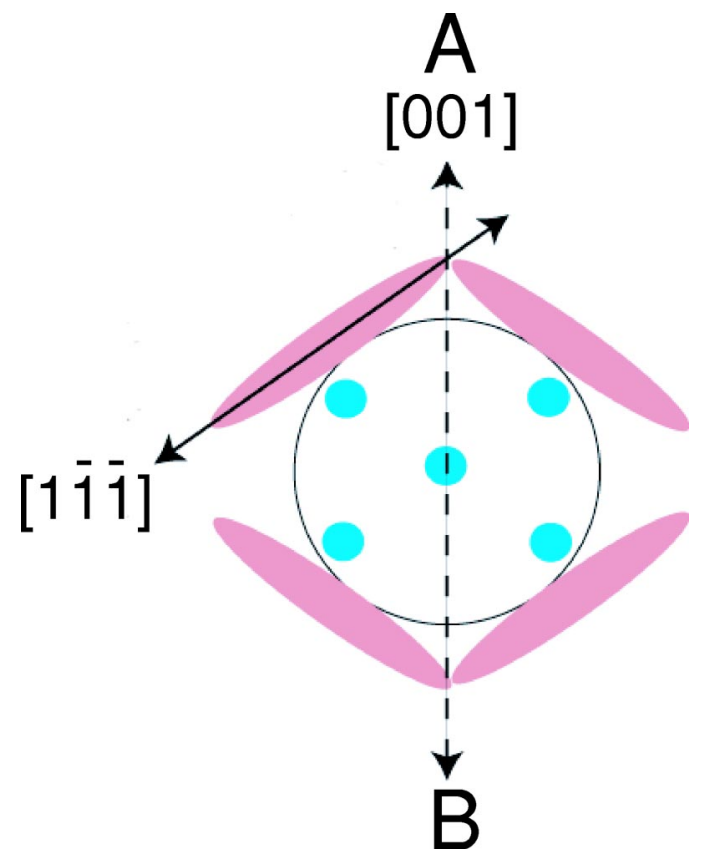

FIG. 10. Schematic diagram of piling up on the (110) surface. The shaded ellipses correspond to the initial formation of pileup. Piling up first occurs at the points at which the $\langle 111\rangle$ directions are tangential to the circular indenter cross section, and the coalescence of the ellipses shows the region where the piling up accumulates in the experiment. The diagram is rotated through $90^{\circ}$ compared to Fig. 9 for direct comparison with Fig. 1(a).

symmetry and for the indentation depth of $4.4 \mathrm{~nm}$, the piling up consists of two sets of two closely separated peaks ("handles") in the in-plane $\langle 111\rangle$ directions. The handles develop along the four corners of the indentation region and begin to merge along the $\langle 001\rangle$ direction as the indentation deepens, to yield a form that resembles more closely the experimental result shown in Fig. 1(b). We suggest that the differences between the experimental results, which show only two peaks, and the simulations is that at the greater depth used in the experiment, the peaks merge according to
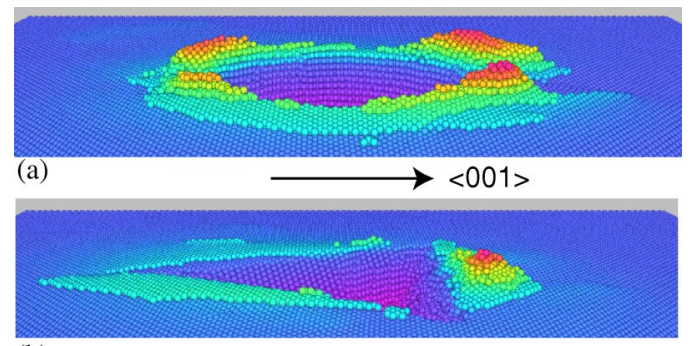

(b)

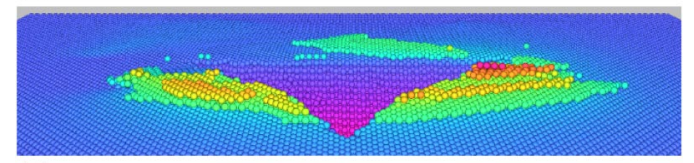

(c)

FIG. 11. MD simulations of pileup of material on the $\{110\}$ face after retraction of the indenter. (a) Paraboloidal interface model; (b) atomistic pyramidal tip in orientation 1; (c) atomistic pyramidal tip in orientation 2 . 


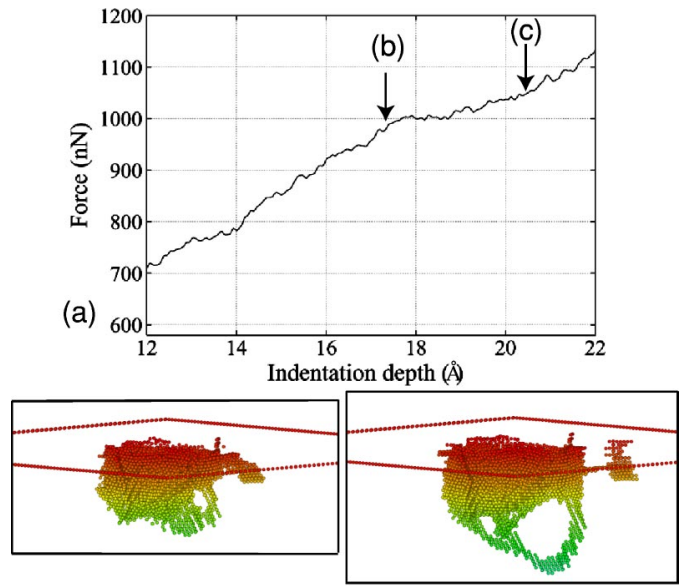

(b)

(c)

FIG. 12. (a) The force-depth curve together with images of the subsurface displaced atoms (b) before and (c) after the emission of the first dislocation loop in the subsurface $\langle 111\rangle$ direction for a 2.5-nm-radius paraboloidal interface. The calculated force-depth curve shows a pop-in between the points corresponding to Figs. 13(b) and 13(c).

the mechanism shown in Fig. 10. Figure 11(b) illustrates the deformation to the surface after extraction of the tip in orientation 1 and shows a pileup of atoms up to 5-6 layers in height close to the hole corresponding to the out-of-plane $\langle 111\rangle$ direction with much less pileup of 1-2 layers on the other sides. As with Fig. 3(a), the smaller pileup areas on the two equivalent sides are separated from the larger pileup by a line perpendicular to the symmetry axis through the center of gravity of the indent image triangle. In Fig. 11(c), for orientation 2 , there is symmetric pileup on two sides of up to three layers in height with a pileup that is only one atomic layer in height on the other side.

Material is moved away from the tip indentation region by two dislocation mechanisms. Dislocation loops are generated that are terminated by edge dislocations in the surface plane, with Burgers vectors of the four in-plane $\langle 111\rangle$ directions. These move out from the tip in these four directions. The dislocation loops that carry material away from the tip indentation region cannot produce any pileup of material. Slip also occurs in the two out-of-plane $\langle 111\rangle$ directions.

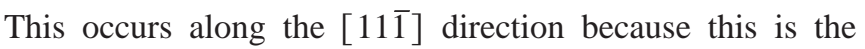
common line to the (101) and (011) planes. Similarly the $(10 \overline{1})$ and $(01 \overline{1})$ planes meet along the $[\overline{1} \overline{1} \overline{1}]$ direction and the pileup is created when cross slip between the $\{110\}$ planes occurs with dislocations propagating in these out-ofplane $\langle 111\rangle$ directions. This mainly occurs in the tip retraction phase of the indentation. The pileup in the case of the spherical indenter clearly illustrates the fact that this mechanism is responsible for the pileup with the four hillocks produced which lie in the four in-plane $\langle 111\rangle$ directions, which are the intersection of the slip planes with the surface.

Figure 12 illustrates one of the mechanisms behind the pop-ins that occur in the experimental force-depth curves. For the parabolic interface model we examine the first pop-in in the calculated force-depth curve. It is clearly seen that this

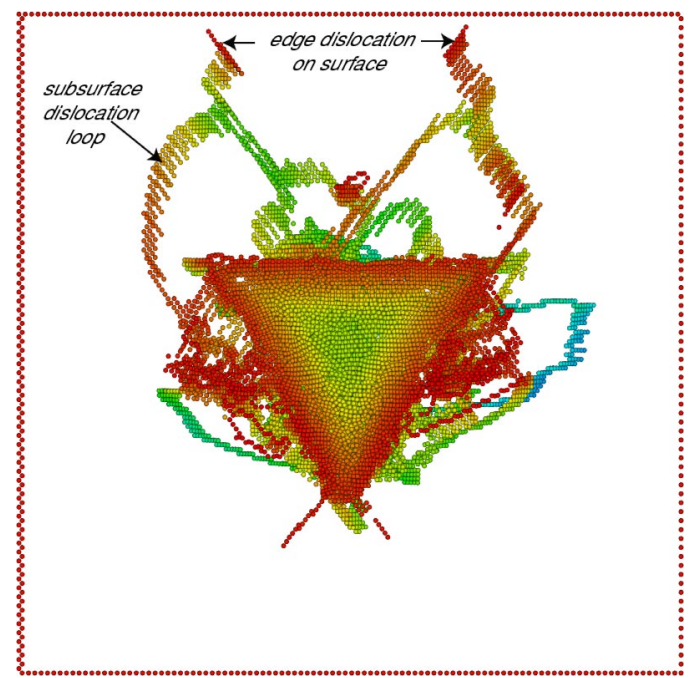

FIG. 13. The dislocation loops formed during the loading part of the indentation for orientation 2 on the $\{110\}$ surface, viewed from directly above the crystal.

corresponds to the emission of the first dislocation loop from the bottom of the tip, which is emitting downwards in the out-of-plane $\langle 111\rangle$ direction along the $\{110\}$ planes. Figure 13 shows the dislocation loops that form and the point at which such loops terminate on the surface in an edge dislocation. There is clear reflection symmetry about the line CD described in Fig. 1(a).

\section{B. Results for the $\{100\}$ plane}

Two rotation angles for the indenter are also used for the $\{100\}$ surface and these are shown in Fig. 14 together with the geometrical arrangement of atoms in the crystal surface. The two different rotation angles will be referred to as orientation 3 and orientation 4, respectively.

When the normal to the pyramidal face is aligned with the $\langle 100\rangle$ direction, orientation 3, then Fig. 15(a) shows that there is only a small pileup along this face compared to the other two faces. This is similar to the result obtained for the $\{110\}$ face, Fig. 11(c). However, for the $\{100\}$ face the pileup is much larger $(1.6 \mathrm{~nm})$ than the $\{110\}$ face due to the way in which the dislocations propagate. When the indenter is aligned as in orientation 4, Fig. 15(b), the normal to one side

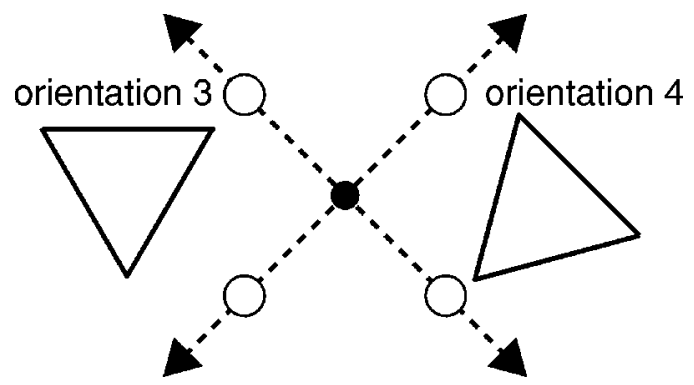

FIG. 14. The two tip orientations used in the simulation of indentation into the $\{100\}$ surface with respect to the layout of atoms in the surface layers. The open circles refer to the positions of atoms in the top layer and the filled circles the second-layer atoms. 


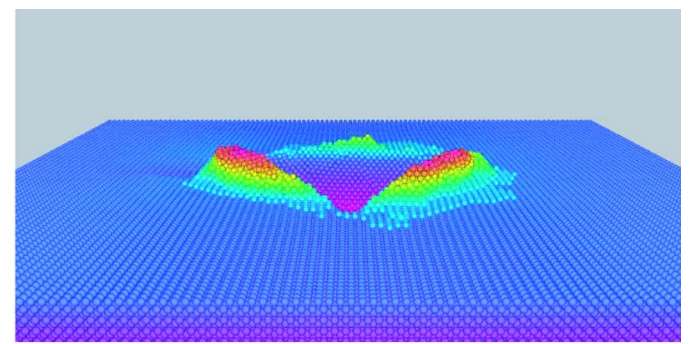

(a)

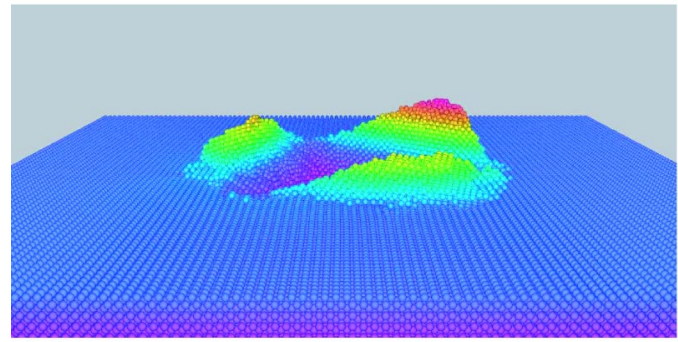

(b)

FIG. 15. MD simulations of pileup of material on the $\{100\}$ face after retraction of the indenter. (a) Pyramidal tip in orientation 3; (b) pyramidal tip in orientation 4 .

of the triangle is aligned along the $\langle 110\rangle$ direction and a larger pileup occurs along this side ( $2.5 \mathrm{~nm}$ in height). Thus although the pileup patterns for $\{110\}$ and $\{100\}$ have a similar shape, they are distinguishable since for the $\{100\}$ face the pileup is much higher for the same indentation force.

For the $\{100\}$ surface there are no in-plane $\langle 111\rangle$ directions and so material cannot be transported away from the hole as for the $\{110\}$ face. Dislocation loops must all propagate in out-of-plane directions. The four out-of-plane $\langle 111\rangle$ directions are also highly symmetric. These facts give rise to the larger pileup seen on this surface. All the dislocation loops that are generated by the indentation process have the potential to cause pileup and the high symmetry of the system means that it is very easy for cross slip to occur. This can clearly be seen in Fig. 16 where the fact that the dislocation loop does not lie in a plane illustrates that cross slip has occurred. For this system cross slip readily occurs on both the indentation and the retraction phases of the indentation process. Figure 16 shows that some dislocation loops terminate at the surface in screw dislocations.

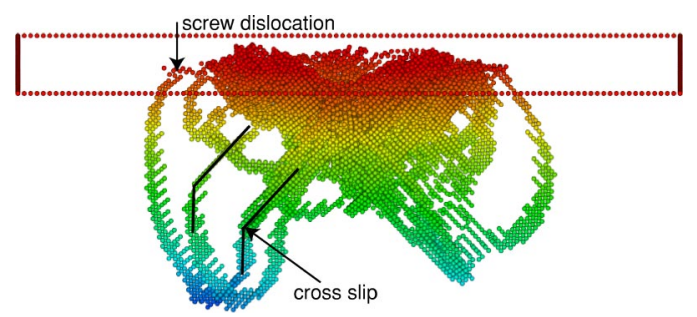

FIG. 16. The dislocation loops below the $\{100\}$ surface. The marked cross slip occurs between planes which intersect the surface at $45^{\circ}$ and those that are vertical.

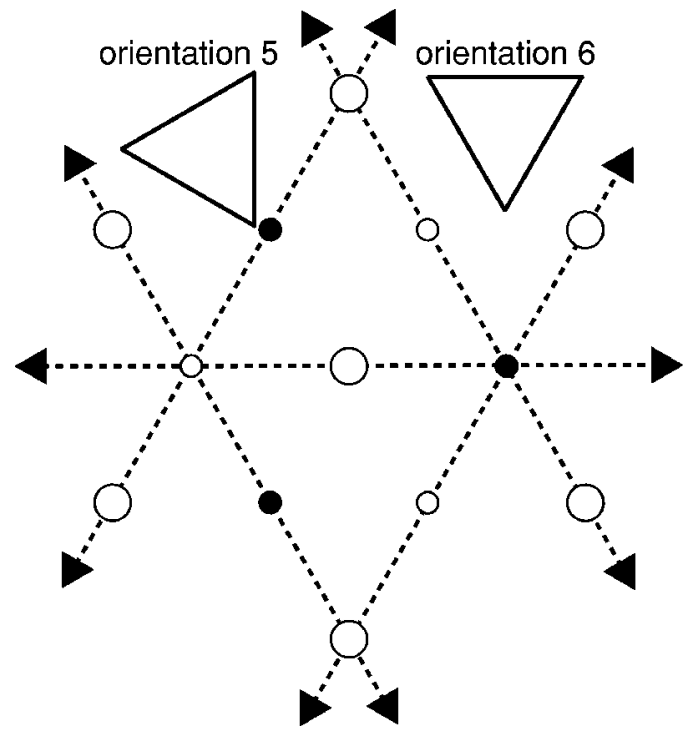

FIG. 17. The two tip orientations used in the simulation of indentation into the $\{111\}$ surface with respect to the layout of atoms in the surface layers. The open circles refer to the positions of atoms in the top layer and the filled circles the second-layer atoms.

\section{Results for the $\{111\}$ plane}

Two rotation angles for the indenter are also used for the $\{111\}$ face and these are shown in Fig. 17 together with the geometrical arrangement of atoms in the crystal surface. The two different rotation angles will be referred to as orientation 5 and orientation 6 , respectively. For the $\{111\}$ surface, the $\{110\}$ slip planes are all inclined to the surface at $35^{\circ}$, i.e., much shallower than the angle at which they intersect with the $\{100\}$ surface. As a result, the piling up is more spread out for this face compared to the other two faces. Both orientations of the indenter are threefold symmetric and so we might expect a completely symmetric piling up. However Fig. 18(a) shows that there is a larger pileup along one face compared to that of the other two faces. Since these pileup heights are so small we speculate that this is a purely statistical effect which will change as the indentation depth increases. For the $\{111\}$ face the piling up is much more spread out than that for the other two faces due to the fact that the slip occurs at shallower angles to the surface. This larger spread-out is also in agreement with the experimental results shown in Fig. 5. The dislocation loops for orientation 6 are shown in Fig. 19 and also exhibit approximate threefold symmetry, in contrast to the reflection symmetry of the loops for the $\{110\}$ surface shown in Fig. 13. For the $\{111\}$ face the piled-up material lies above the region of the crystal where the dislocation loops have formed.

\section{Force-depth curves and their analysis}

Figure 20 shows that the force on the indenter for the $\{110\}$ surface as a function of depth is very similar regardless of tip orientation. Similar results and maximum forces are obtained for indentation on the $\{100\}$ and $\{111\}$ faces. The maximum forces for the three faces are $2.13 \mu \mathrm{N}$ for $\{100\}$, $2.1 \mu \mathrm{N}$ for $\{111\}$, and $2.04 \mu \mathrm{N}$ for $\{110\}$. The contact depth 


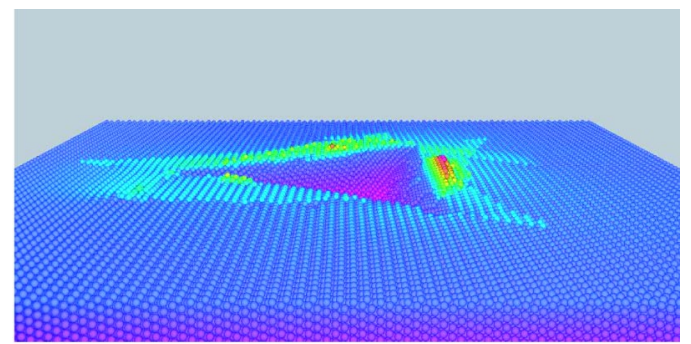

(a)

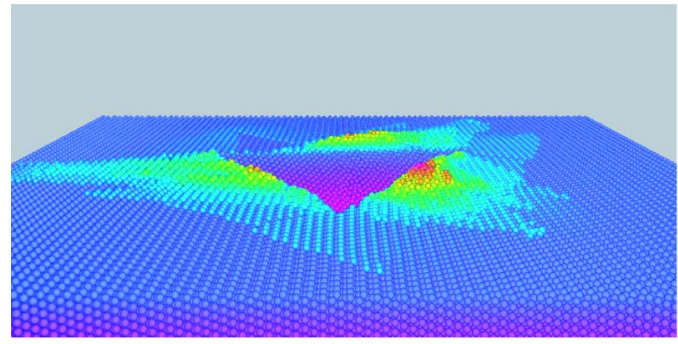

(b)

FIG. 18. MD simulations of pileup of material on the $\{111\}$ face after retraction of the indenter. (a) Pyramidal tip in orientation 5; (b) pyramidal tip in orientation 6 .

$h_{c}$ is almost the same for all three directions, amounting to $h_{c}=3.5 \mathrm{~nm}$. For this contact depth a contact area of $65 \mathrm{~nm}^{2}$ is derived. By dividing the maximum forces by this area, contact pressures (nanohardness values) of $32.5 \mathrm{GPa}, 32.3$ $\mathrm{GPa}$, and $31.4 \mathrm{GPa}$ are obtained. There is thus only a $3 \%$ difference in the calculated hardnesses of the three faces. The small hardness dependency on orientation can to some extent be due to the fact that variations of the true contact area owing to different piling up is not considered here. However, the hardness values are four times higher than the smallest depth experimental values. It is known that the experimentally measured contact pressure increases with decreasing indentation depth, as shown in Fig. 8. The simulations also show that the hardness also decreases with increasing depth.

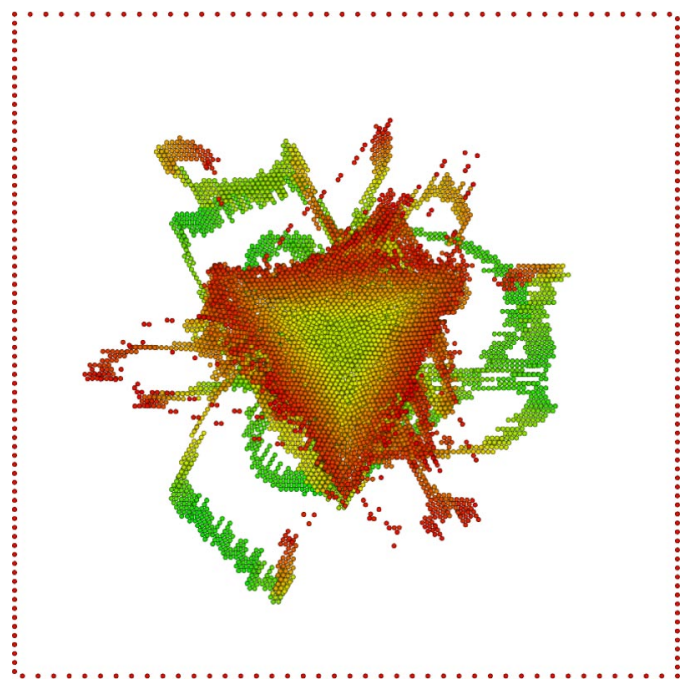

FIG. 19. The dislocation loops on and under the $\{111\}$ plane (orientation 6) viewed from directly above the indenter.

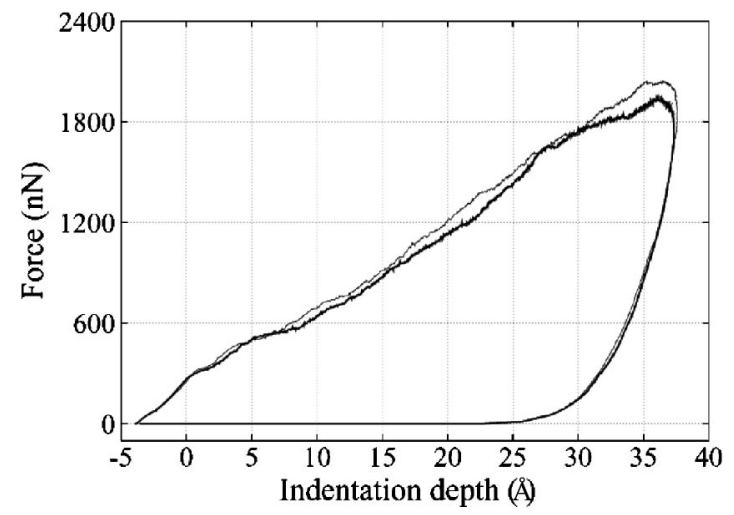

FIG. 20. The calculated force-depth curves for the two orientations of the pyramidal diamond indenter. A similar curve is obtained for the paraboloidal interface model except that for the atomistic tip, the maximum depth is less than the displacement of the fixed top layers due to a $4 \%$ tip compression.

Since the cross-sectional area of the indenter is proportional to the square of the indentation depth, for constant hardness the force would vary parabolically with indentation depth. Figure 20 clearly shows that this is not the case and it is almost linear. A similar linear behavior is observed experimentally for small indentation depths up to about $8 \mathrm{~nm}$, in Fig. 7(b). At the moment we have no satisfactory explanation for this almost linear dependence. A similar discrepancy arises with the calculated indentation modulus. The indentation modulus $E_{r}$ was derived from the unloading part of the load-depth curve (Fig. 20) using the Hertzian model, ${ }^{21,22}$ giving a value $E_{r}=600 \mathrm{GPa}$. This is a factor-of-3 higher than the Young's modulus of polycrystalline, i.e., quasiisotropic iron of about $200 \mathrm{GPa}$.

\section{DISCUSSION AND CONCLUSION}

Despite the differences in length scales inherent in the experiments and simulations, there is remarkable agreement between the two sets of the piling-up patterns. The nanoindentation technique has clearly demonstrated the different mechanical behavior that arises when different crystal faces are indented. The planes in which slip occurs are of the $\{110\}$ family and the simulations have shown that the piling up occurs as a result of cross slip between planes of this family. When these planes intersect the surface at a shallow angle, pileup is extended away from the hole so that the $\{111\}$ surface has a shallower pileup than the $\{100\}$. The anisotropies in the piling up patterns on some faces are so strong that the nanoindentation technique could even be used as a quick method for determining crystal orientation. The quantitative agreement between the mechanical properties determined from the simulation curves and the experiment is less impressive due to the very small system size that simulation is currently able to handle. Better agreement here would be expected with the advent of even more powerful computers and more realistic simulations. Nonetheless, a correlation has been shown between the first emission of dislocation loops and the pop-ins in the force-depth curves. The fact that the calculated "nanohardness" was about four 
times larger than the contact pressure measured in experiment is not so surprising since the experiments themselves show that nanohardness itself is not a constant value and shows a large increase as the indentation depth is reduced. The ability of the simulations to explain the main experimental features for a perfect single element crystalline structure such as iron gives confidence that the techniques can be used to analyze nanoindentation of more complex materials and thin-film structures in which the material displacement mechanisms will be even more involved.

\section{ACKNOWLEDGMENTS}

We would like to thank Bruce King of Newcastle University, Australia for providing the samples and helpful discussions and Mrs. Karola Kubica for help in sample preparation. The work was also funded in part by EPSRC Grant No. GR/R18581 and e-Science Grant No. GR/R67699. CPU time was provided by the University of Manchester Regional Computer Center. The experimental work was funded by an HPS-N Project from the state of Brandenburg. We also thank Steve Roberts and Ronald Ries for useful discussions.
${ }^{1}$ W. C. Olvier and G. M. Pharr, J. Mater. Res. 7, 1564 (1992).

${ }^{2}$ U. Landmann, W. D. Luedtke, N. A. Burnham, and R. J. Colton, Science (Washington, DC, U.S.) 248, 454 (1990).

${ }^{3}$ J. D. Kiely and J. E. Houston, Phys. Rev. B 57, 12588 (1998).

${ }^{4}$ S. G. Corcoran, R. J. Colton, E. T. Lilleodden, and W. W. Gerberich, Phys. Rev. B 55, R16 057 (1997).

${ }^{5}$ O. R. de la Fuente, J. A. Zimmerman, M. A. Gonzalez, J. de la Figuera, J. C. Hamilton, W. W. Pai, and J. M. Rojo, Phys. Rev. Lett. 88, 036101 (2002).

${ }^{6}$ A. Richter, R. Ries, R. Smith, M. Henkel, and B. Wolf, Diamond Relat. Mater. 9, 170 (2000).

${ }^{7}$ D. Christopher, A. Richter, and R. Smith, Nanotechnology 12, 372 (2001).

${ }^{8}$ R. Astala, R. M. Niemenen, and T. Heine, Phys. Rev. B 61, 2973 (2000).

${ }^{9}$ R. Perez, M. C. Payne, and A. D. Simpson, Phys. Rev. Lett. 75, 4748 (1995).

${ }^{10}$ C. Kelchner, S. J. Plimpton, and J. C. Hamilton, Phys. Rev. B 58, 11085 (1998).

${ }^{11}$ D. Christopher, S. D. Kenny, R. Smith, A. Richter, R. Ries, and B.
Wolf, in Multiscale Phenomena in Materials Experiments and Modeling Related to Mechanical Behavior, Proc. MRS Spring Symposium W (2003) (in press).

${ }^{12}$ A. V. Kulkarni, J. T. Wyrobek, Z. Qian, J. M. Siversten, and J. Judy (unpublished).

${ }^{13}$ B. Bushan, A. V. Kulkarni, W. Bonin, and J. T. Wyrobek, Philos. Mag. A 74, 1117 (1996).

${ }^{14}$ B. Wolf, Cryst. Res. Technol. 35, 377 (2000).

${ }^{15}$ F. Kick, The Law of Proportional Resistance and its Applications (Felix-Verlag, Berlin, 1885).

${ }^{16}$ D. W. Brenner, Phys. Rev. B 42, 9458 (1990).

${ }^{17}$ D. W. Brenner, Phys. Rev. B 46, 1948 (1992).

${ }^{18}$ J. F. Ziegler, J. P. Biersack, and U. Littmark, The Stopping and Range of Ions in Solids (Pergamon, London, 1985).

${ }^{19}$ D. Christopher, Ph.D. thesis, Loughborough University, 2001.

${ }^{20} \mathrm{R}$. Smith, Atomic and Ion Collisions in Solids and at Surfaces (Cambridge University, Cambridge, England, 1997).

${ }^{21}$ K. L. Johnson, Contact Mechanics (Cambridge University, Cambridge, England, 1985).

${ }^{22}$ B. Wolf, M. Swain, M. Kempf, and P. Paufler, J. Mater. Sci. 35, 723 (2000). 